\title{
Indefinite Harmonic Forms and Gauge Theory
}

\author{
M. Nakashima \\ Department of Physics, University of California, Los Angeles, CA 90024, USA
}

\begin{abstract}
Indecomposable representations have been extensively used in the construction of conformal and de Sitter gauge theories. It is thus noteworthy that certain unitary highest weight representations have been given a geometric realization as the unitary quotient of an indecomposable representation using indefinite harmonic forms [RSW]. We apply this construction to $S U(2,2)$ and the de Sitter group. The relation is established between these representations and the massless, positive energy representations of $S U(2,2)$ obtained in the physics literature. We investigate the extent to which this construction allows twistors to be viewed as a gauge theory of $S U(2,2)$. For the de Sitter group, on which the gauge theory of singletons is based, we find that this construction is not directly applicable.
\end{abstract}

\section{Introduction}

Representations of space-time symmetry groups play a fundamental role in physics. In particular, minimal energy, indecomposable representations [A], in which the physical sector is realized on a unitary subquotient, have been extensively used in the construction of Poincaré, de Sitter, and conformal gauge theories ([AFFS, BFH1, 2, F, FF1-6, FPS, Ha], and references therein). Especially interesting is the recent progress in singletons ([FF1-6]), a theory which has its origins in the work of Dirac [D2]. It is thus extremely interesting that in the recent mathematical literature, certain representations of this type have been given a very elegant geometric realization in terms of indefinite harmonic forms [RSW]. Several physical applications of this indefinite harmonic construction are immediately suggested, and these possibilities will be investigated in this note. First, let us introduce the representation in question.

Recall that the unitary irreducible representations of semisimple Lie groups can be divided into two classes: the regular representations, which occur in the Plancherel decomposition of $L_{2}(G)$, and the singular representations, which do not. More precisely, the representations treated in [RSW] are highest weight representations lying in the analytic continuation of the holomorphic discrete series (which we take to include those discrete points known as the "Wallach set"). Basically, the construction is the following. Given a Lie group $G$, a subgroup $H$ 
is chosen such that $G / H$ is a pseudo-Hermitian symmetric space. For a given representation of $H$ on a vector space $V, G$ acts on the Dolbeault cohomology of the associated homogeneous vector bundle, and this action is unitarized by passing to $L_{2}$ indefinite harmonic forms. However, in order for this construction to work, the space $G / H$ must satisfy a very restrictive requirement. Namely, if $K$ is maximal compact in $G$, and, replacing $K$ by a suitable conjugate if necessary, if $H \cap K$ is maximal compact in $H$, it is required that $G / H \cap K$ carry an invariant complex structure such that both $G / H \cap K \rightarrow G / H$ and $G / H \cap K \rightarrow G / K$ are holomorphic.

As an example in [RSW], this construction is applied to the indefinite unitary groups, $U(p, q)$, realizing most of the unitarizable highest weight modules. Several possible applications to physics are also noted. A strong formal analogy is pointed out between the indefinite harmonic construction and Gupta-Bleuler indefinite metric quantization [W1,2]; a relationship between the case $G=U(2,2)$, $H=U(1) \times U(1,2)$, and twistor theory is implied; and the indication is given that the Dirac singletons are realized in the context of their paper on the space $S p(2, \mathbb{R}) / U(1,1)[\mathrm{RSW}$, Appendix $]$.

We will investigate these possibilities by first working out in detail the indefinite harmonic construction for $S U(2,2)$. This will serve to introduce the construction as well as to exhibit the relation to the massless representations as obtained in the physics literature. Then, after briefly reviewing the most basic gauge theory based on an indecomposable representation, we will see how the indefinite harmonic construction allows twistors to be viewed as a gauge theory of the conformal group. We will also attempt to construct a Gupta-Bleuler triplet in the context of twistors. Finally, in the hope of obtaining a geometric realization of the Dirac singletons, we will attempt to apply the indefinite harmonic construction to the de Sitter group. However, we will find that for this group or any of its covers, the previously mentioned holomorphic condition cannot be met.

Let us now introduce some notation. Let $G$ be a connected, reductive Lie group, and $\tau$ an involutive automorphism of $G$. Let $H=\left(G^{\tau}\right)^{0}$ denote the identity component of the fixed point set of $\tau$. Choose a Cartan involution $\theta$ that commutes with $\tau$. Let $K=G^{\theta}$ denote the fixed point set of $\theta$. Set $M=G^{\tau \theta}$ and $L=H \cap K$. Denote the respective real Lie algebras of $G, H, K, M, L$ by $\mathfrak{g}_{0}, \mathfrak{h}_{0}, \mathfrak{l}_{0}, \mathrm{~m}_{0}, \mathrm{l}_{0}$. The \pm 1 eigenspace decompositions will be written as

$$
\begin{array}{ll}
\mathfrak{g}_{0}=\mathfrak{h}_{0}+\mathfrak{q}_{0} & \text { under } \tau, \text { and } \\
\mathfrak{g}_{0}=\mathfrak{F}_{0}+\mathfrak{p}_{0} & \text { under } \theta .
\end{array}
$$

Complexifications will be denoted by dropping the subscript. The almost complex structure on $G / H\left(G / K\right.$ respectively) is given by $\operatorname{ad}_{\mathfrak{q}} \zeta\left(\operatorname{ad}_{\mathfrak{p}} \eta\right)$, where $\zeta(\eta)$ is a central element of $\mathfrak{h}_{0}\left(\mathfrak{f}_{0}\right)$. The holomorphic and anti-holomorphic tangent spaces of $G / H$ are given respectively by

$$
\mathfrak{q}_{+}=\{\xi \in \mathfrak{q}:[\zeta, \xi]=i \xi\}, \quad \mathfrak{q}_{-}=\{\xi \in \mathfrak{q}:[\zeta, \xi]=-i \xi\}
$$

(likewise for $\mathfrak{p}_{+}$and $\mathfrak{p}_{-}$). 


\section{II. $S U(2,2)$}

As a way of introducing the indefinite harmonic construction of [RSW], we will work out the case $G=S U(2,2)$ in detail. First, we will examine the space $G / H$, explicitly verifying the holomorphic condition and giving a local coordinate parametrization of the $G$-action. Next, we will give an explicit construction of the holomorphic line bundles induced by one dimensional representations of $H$, and then we will outline the unitary construction. At the end of this section, we will relate the ladder representations as realized in the indefinite harmonic construction to the positive energy representations as obtained in the physics literature.

$A$. The Space $G / H$. Let $G=S U(2,2)$. The requirement that $G / H$ be a pseudoHermitian symmetric space requires $H=S(U(1,1) \times U(1,1)), H=S(U(1) \times U(1,2))$, or $H=S(U(2,1) \times U(1))[\mathrm{Sr}]$. In order to satisfy the afore-mentioned holomorphic requirement, $H$ is further restricted to be $S(U(1) \times U(1,2))$ or $S(U(2,1) \times U(1))$ [RSW, 2.22]. However, to avoid redundancy, we will work with $H=S(U(1) \times$ $U(1,2)$ ), making note of $H=S(U(2,1) \times U(1))$ when necessary. Let us now verify explicitly that $G / H=S(2,2) / S(U(1) \times U(1,2))$ does satisfy the holomorphic condition. Thus

$$
\begin{aligned}
\mathfrak{g}_{0} & =\mathfrak{s u}(2,2), \\
\mathfrak{h}_{0} & =\mathfrak{s}(\mathfrak{u}(1)+\mathfrak{u}(1,2)), \\
\mathfrak{I}_{0} & =\mathfrak{s}(\mathfrak{u}(2)+\mathfrak{u}(2)), \\
\mathfrak{m}_{0} & =\mathfrak{s}(\mathfrak{u}(1)+\mathfrak{u}(1,2)), \\
\mathfrak{I}_{0} & =\mathfrak{s}(\mathfrak{u}(1)+\mathfrak{u}(1)+\mathfrak{u}(2)) .
\end{aligned}
$$

Parametrize $\mathfrak{g}_{0}$ in the following manner:

$$
\mathfrak{g}_{0}=\left\{\left[\begin{array}{cccc}
i \theta_{1} & a & c & d \\
-\bar{a} & i \theta_{2} & e & f \\
\bar{c} & \bar{e} & i \theta_{3} & b \\
\bar{d} & \bar{f} & -\bar{b} & i \theta_{4}
\end{array}\right]: \theta_{i} \in \mathbb{R}, a, \ldots f \in \mathbb{C} ; \Sigma \theta_{i}=0\right\} .
$$

Then

$$
\begin{aligned}
\mathfrak{h}_{0} & =\left\{\left[\begin{array}{cccc}
i \theta_{1} & 0 & 0 & 0 \\
0 & i \theta_{2} & e & f \\
0 & \bar{e} & i \theta_{3} & b \\
0 & \bar{f} & -\bar{b} & i \theta_{4}
\end{array}\right]: \theta_{i} \in \mathbb{R}, b, e, f \in \mathbb{C} ; \Sigma \theta_{i}=0\right\} \\
\mathfrak{f}_{0} & =\left\{\left[\begin{array}{cccc}
i \theta_{1} & a & 0 & 0 \\
-\bar{a} & i \theta_{2} & 0 & 0 \\
0 & 0 & i \theta_{3} & b \\
0 & 0 & -\bar{b} & i \theta_{4}
\end{array}\right]: \theta_{i} \in \mathbb{R}, a, b \in \mathbb{C} ; \Sigma \theta_{i}=0\right\}
\end{aligned}
$$




$$
\begin{aligned}
& \mathfrak{q}_{0}=\left\{\left[\begin{array}{cccc}
0 & a & c & d \\
-\bar{a} & 0 & 0 & 0 \\
\bar{c} & 0 & 0 & 0 \\
\bar{d} & 0 & 0 & 0
\end{array}\right]: a, c, d \in \mathbb{C}\right\} \\
& \mathfrak{p}_{0}=\left\{\left[\begin{array}{cccc}
0 & 0 & c & d \\
0 & 0 & e & f \\
\bar{c} & \bar{e} & 0 & 0 \\
\bar{d} & \bar{f} & 0 & 0
\end{array}\right]: c, d, e, f \in \mathbb{C}\right\} \\
& \mathfrak{m}_{0}=\left\{\left[\begin{array}{cccc}
i \theta_{1} & 0 & c & d \\
0 & i \theta_{2} & 0 & 0 \\
\bar{c} & 0 & i \theta_{3} & b \\
\bar{d} & 0 & -\bar{b} & i \theta_{4}
\end{array}\right]: b, c, d \in \mathbb{C}, \theta_{i} \in \mathbb{R}, \Sigma \theta_{i}=0\right\} \\
& \mathfrak{l}_{0}=\left\{\left[\begin{array}{cccc}
i \theta_{1} & 0 & 0 & 0 \\
0 & i \theta_{2} & 0 & 0 \\
0 & 0 & i \theta_{3} & b \\
0 & 0 & -\bar{b} & i \theta_{4}
\end{array}\right]: b \in \mathbb{C}, \theta_{i} \in \mathbb{R}, \Sigma \theta_{i}=0\right\} .
\end{aligned}
$$

The central elements giving the almost complex structure (unique up to sign since $G$ is simple) on $G / H$ and $G / K$ respectively are

$$
\zeta=-\frac{1}{4}\left[\begin{array}{cccc}
-3 i & 0 & 0 & 0 \\
0 & i & 0 & 0 \\
0 & 0 & i & 0 \\
0 & 0 & 0 & i
\end{array}\right] \text { and } \eta=\frac{1}{2}\left[\begin{array}{cccc}
i & 0 & 0 & 0 \\
0 & i & 0 & 0 \\
0 & 0 & -i & 0 \\
0 & 0 & 0 & -i
\end{array}\right] \text {. }
$$

Direct calculation gives

$$
\begin{aligned}
\mathfrak{q}_{+}= & \left\{\left[\begin{array}{llll}
0 & a & c & d \\
0 & 0 & 0 & 0 \\
0 & 0 & 0 & 0 \\
0 & 0 & 0 & 0
\end{array}\right]: a, c, d \in \mathbb{C}\right\} \\
\mathfrak{p}_{+}= & \left\{\left[\begin{array}{llll}
0 & 0 & c & d \\
0 & 0 & e & f \\
0 & 0 & 0 & 0 \\
0 & 0 & 0 & 0
\end{array}\right]: c, d, e, f \in \mathbb{C}\right\} .
\end{aligned}
$$

A necessary and sufficient condition for both $G / L \rightarrow G / K$ and $G / L \rightarrow G / H$ to be holomorphic is

$$
\left[\mathfrak{p} \cap \mathfrak{q}_{+},\left[\mathfrak{p} \cap \mathfrak{q}_{+}, \mathfrak{l} \cap \mathfrak{q}_{-}\right]\right]=0 \quad[\mathrm{RSW}, 2.21] .
$$

Direct calculation shows this to hold.

Now having verified that $S U(2,2) / S(U(1) \times U(1,2))$ is an appropriate space for $S U(2,2)$, let us note a few of its properties. As a pseudo-Hermitian symmetric space, $G / H$ has a $C^{\infty}$ fibration $\pi: G / H \rightarrow K / L$, where $K / L$ is the maximal compact subvariety. A typical fiber is $M / L$, which has the structure of a Hermitian bounded 
symmetric domain [Sr]. In our case,

$$
K / L=S(U(2) \times U(2)) / S(U(1) \times U(1) \times U(2)) \approx \mathbb{P} \mathbb{C}^{1},
$$

and $\operatorname{dim}_{\mathbb{C}} K / L=1$. Furthermore, our $G / H$ is an open $S U(2,2)$ orbit in the complex flag manifold, $S L(4, \mathbb{C}) / P$, where $P$ is the parabolic subgroup

$$
P=\left\{\left[\begin{array}{cccc}
* & 0 & 0 & 0 \\
* & * & * & * \\
* & * & * & * \\
* & * & * & *
\end{array}\right] \in S L(4, \mathbb{C})\right\}
$$

$S L(4, \mathbb{C}) / P$ is equivalent to $\mathbb{P} \mathbb{C}^{3}$, and by considering $G / H$ as an open subset of $P \mathbb{C}^{3}$, we can explicitly exhibit the action of $G$ in terms of local coordinates. Let

$$
\zeta=\left[\zeta^{0}, \zeta^{1}, \zeta^{2}, \zeta^{3}\right]
$$

be homogeneous coordinates on $\mathbb{P C ^ { 3 }}$, and let

$$
U^{i}=\left\{\zeta \in \mathbb{P} \mathbb{C}^{3}: \zeta^{i} \neq 0\right\}, \quad i=0,1 .
$$

$S L(4, \mathbb{C})$ acts on the homogeneous coordinates by right matrix multiplication, and this is easily written in terms of local coordinates. In $U^{0}$, local coordinates are given by

$$
z=\left[z^{1}, z^{2}, z^{3}\right] \quad \text { where } \quad z^{i}=\frac{\zeta^{i}}{\zeta^{0}}
$$

Write $g \in S L(4, \mathbb{C})$ as

$$
g=\left[\begin{array}{l|l}
A & C \\
\hline B & D
\end{array}\right]
$$

Then $z g=(z D+C / z B+A)$.

Note that $P$ is precisely the stabilizer of $z=[0,0,0]$. Likewise, in $U^{1}$ we have local coordinates

$$
w=\left[w^{1}, w^{2}, w^{3}\right] \quad \text { where } \quad w^{1}=\frac{\zeta^{0}}{\zeta^{1}}, \quad w^{i}=\frac{\zeta^{i}}{\zeta^{1}}, \quad i=2,3 .
$$

If we impose the $G$-invariant indefinite Hermitian form

$$
\Phi \text { with signature }(+1,+1,-1,-1) \text {, }
$$

then space $S U(2,2) / S(U(1) \times U(1,2))$ can be described as those $\zeta$ positive under $\Phi$. This is precisely the $S U(2,2)$ orbit of $\zeta=[1,0,0,0]$. Note that in the case of $H=S(U(2,1) \times U(1))$, we merely have a different open $S U(2,2)$ orbit, namely those $\zeta$ negative under $\Phi$.

The Killing form on $\mathfrak{g}$ induces a $G$-invariant pseudo-Hermitian Kähler metric on $G / H$. It is essentially an indefinite version of the Fubini-Study metric, and on $U^{0}$ it has the following local expression:

$$
g_{i \bar{j}}=\frac{1}{a^{2}}\left(a I_{i \bar{j}}+I_{i \bar{k}} \bar{z}^{\bar{k}} z^{1} I_{1,}\right)
$$


where $\operatorname{diag}\left(I_{\imath \jmath}\right)=(+1,-1,-1)$ and $a=\left(1+\bar{z}^{-} I_{l \jmath} z^{j}\right)$. The inverse $g^{i \bar{l}}$, where $g_{i j}-g^{j k}=\delta_{i}^{k}$, is given by

and

$$
g^{\bar{l} j}=a\left(I^{\bar{j}}+\bar{z}^{\bar{i}} z^{j}\right)
$$

$$
\operatorname{det}\left(g_{i \bar{j}}\right)=\frac{1}{a^{4}}
$$

B. Holomorphic Line Bundles on $G / H$. The next step in the indefinite harmonic construction is to take a unitary representation of $H$ on a vector space $V$ and then form the $C^{\infty}$ homogeneous vector bundle, $\mathbf{V} \rightarrow G / H$, where $\mathbf{V}=G \times_{H} V$. As the ladder representations of $G$ are obtained from one-dimensional representations of $H$, let us from this point on restrict our attention to unitary characters of $H$. The resulting line bundle can be given a holomorphic structure, and this can be seen in the following way. First, consider $G / H$ as imbedded in the complex flag manifold, $G_{\mathbb{C}} / P=\mathbb{P} \mathbb{C}^{3}$. Pick $x \in G_{\mathbb{C}} / P$. There exists a holomorphic function

$$
s^{i}: U^{i} \rightarrow G_{\mathbb{C}} \text { such that } x s^{i}(\zeta)=\zeta .
$$

On $U^{i} \cap U^{j}$, define $s^{i j}$ by

$$
s^{i j}(\zeta)=s^{i}(\zeta) s^{j}(\zeta)^{-1}
$$

Note $s^{i j}(\zeta) \in P$. A given character $\lambda$ of $H$, trivial on $U(1,2)$, extends uniquely to a character of $P$. With $h^{i j}=\lambda \cdot s^{i j}$ as transition functions, we thus obtain a holomorphic line bundle $G_{\mathbb{C}} \times{ }_{P} V$ over $G_{\mathbb{C}} / P$. Restricting to $G / H$ gives the holomorphic structure of $G \times{ }_{H} V$.

If our inducing character corresponds to the integer $m$, the transition function on $U^{0} \cap U^{1}$ is

$$
h^{10}(\zeta)=\left[\frac{\zeta^{0}}{\zeta^{1}}\right]^{m} .
$$

Thus, we have $\mathbf{V}=\mathbf{H}^{m}$, where $\mathbf{H}$ is the hyperplane bundle and $m$ is the integer corresponding to the inducing character.

C. The Unitary Construction. Given a unitary representation of $H$ on a vector space $V$, there is under certain circumstances a natural representation of $G$ on the Dolbeault cohomology $H^{s}(G / H, \mathbf{V})$, where $s=\operatorname{dim}_{\mathbb{C}} K / L$. However, as the natural $G$-invariant metric on $G / H$ is not positive definite, this representation must be unitarized. We will just sketch the idea, and for a more detailed summary, one may consult [W1] or [W2].

Note first that the $G$-invariant indefinite Kähler metric together with the Hermitian fiber metric on $\mathbf{V} \rightarrow G / H$, defines the Kodaira-Hodge star operator

$$
*: E^{p, q}(G / H, \mathbf{V}) \rightarrow E^{n-p, n-q}\left(G / H, \mathbf{V}^{*}\right),
$$

where $E^{a, b}(G / H, \mathbf{V})$ is the space of $C^{\infty} \mathbf{V}$-valued $(a, b)$ forms on $G / H, n=\operatorname{dim}_{\mathbb{C}} G / H$, and $\mathbf{V}^{*} \rightarrow G / H$ is the dual bundle. Thus there is a $G$-invariant indefinite Hermitian inner product

$$
\langle\phi, \psi\rangle=\int_{G / H} \phi(x) \bar{\wedge} * \psi(x) d(x H)
$$


where $\bar{\lambda}$ denotes exterior product followed by contraction of $V$ against $V^{*}$. The $\bar{\partial}$ and $\bar{\partial}^{*}$ operators are defined as in the positive definite case, and the definition is made that a form

$$
\phi \in E^{p, q}(G / H, \mathbf{V}) \text { is harmonic if } \bar{\partial} \phi=0 \text { and } \bar{\partial}^{*} \phi=0 .
$$

However, the indefinite case differs from the positive definite case in that a Dolbeault cohomology class no longer has a unique harmonic representative. Therefore, [RSW] consider a positive definite metric which is not $G$-invariant, but of bounded distortion, and this gives rise to a Hilbert space $L_{2}^{p, q}(G / H, \mathbf{V})$, the completion of the space of $C_{0}^{\infty}$ forms in $E^{p, q}(G / H, \mathbf{V})$ relative to the positive definite metric. This space still carries the $G$-invariant indefinite Hermitian metric, and under the appropriate conditions, each Dolbeault cohomology class has a unique "special" harmonic representative in $L_{2}^{p, q}(G / H, \mathbf{V})$, that is a representative form that is both harmonic relative to the $G$-invariant indefinite metric and of a "special" form [RSW, 7.23]. Thus the basic $L_{2}$ harmonic space is taken to be

$$
\mathscr{H}_{2}^{s}(G / H, \mathbf{V})=\left\{\phi \in L_{2}^{0, s}(G / H, \mathbf{V}): \bar{\partial} \phi=0 \text { and } \bar{\partial}^{*} \phi=0 \text { as distributions }\right\}
$$

(one can use forms with distributional coefficients as well as $C^{\infty}$ ).

Now consider the natural map

$$
\mathscr{H}_{2}^{s}(G / H, \mathbf{V}) \rightarrow H^{s}(G / H, \mathbf{V}),
$$

which sends each form to its corresponding Dolbeault class. Under certain conditions, the $G$-invariant Hermitian metric $\langle$,$\rangle is positive semi-definite on$ $\mathscr{H}_{2}^{s}(G / H, \mathbf{V})$, and the kernel of the map corresponds to the null space of $\langle$, (2.4). The null space is a closed, invariant subspace, and the quotient

$$
\overline{\mathscr{H}}_{2}^{\mathrm{s}}(G / H, \mathbf{V})=\overline{\mathscr{H}}_{2}^{\mathrm{s}}(G / H, \mathbf{V}) /(\text { null space of }\langle,\rangle \text { ) }
$$

forms a Hilbert space that carries a unitary representation of $G$. As $\left(\mathfrak{g}_{0}, K\right)$ modules, $\overline{\mathscr{H}}_{2}^{s}(G / H, \mathbf{V})_{K}$ is isomorphic to $H^{s}(G / H, \mathbf{V})_{K}$, and $\overline{\mathscr{H}}_{2}^{s}(G / H, \mathbf{V})$ gives a unitarization of the action of $G$ on the Dolbeault cohomology $H^{s}(G / H, \mathbf{V})$.

D. Ladder Representations. As already mentioned, certain conditions must be met in order for the above construction to work. In particular, assuming that $G / H$ satisfies the holomorphic condition, the inducing representation of $H$ must satisfy a " $L_{2}$-condition" [RSW: 13.12]. Applying this condition to representations of $S U(2,2)$ induced by one dimensional representations of $H$, be inducing character of $H$ must satisfy

$$
m \leqq-3, \quad m \in \mathbb{Z},
$$

where $m$ is as in (2.2). This produces the highest weight ladder representations, and the duals are lowest weight representations. A change of basis shows that they correspond to globalizations of the positive energy modules

$$
D(j+1, j, 0) ; \quad 2 j \geqq 1,2 j \in \mathbb{Z},
$$

described in the physics literature ([F,BFH1]). Namely, we have massless representations (i.e. representations that restrict to massless, unitary irreducible 
representations of the Poincaré group) of positive helicity where

$$
E=-\frac{m}{2}, \quad j=-\frac{m}{2}-1 \text {. }
$$

The massless representations of negative helicity, $D(j+1,0, j)$, can be constructed in an analogous manner by working instead with $G / H=S U(2,2) / S(U(2,1) \times U(1))$.

We will now examine the subspace structure of the $\left(g_{0}, K\right)$ module $\mathscr{H}_{2}^{s}(G / H, \mathbf{V})_{K}$. As $\overline{\mathscr{H}}_{2}^{s}(G / H, \mathbf{V})_{K}$ is an irreducible highest weight module, it is the irreducible quotient of the highest weight module $\mathscr{H}_{2}^{s}(G / H, \mathbf{V})_{K}$ by its maximal invariant submodule. The subspace structure of all such modules for $S U(2,2)$ has been determined by Jakobsen [J]. Using his results, which utilize Weyl equivalence, we find that the null space corresponds, in the notation of $[\mathrm{F}]$ and $[\mathrm{BFH} 1]$, to

$$
D\left(j+2, j-\frac{1}{2}, \frac{1}{2}\right) .
$$

\section{Gauge Theory and Twistors}

As mentioned in the introduction, indecomposable representations have been used extensively in the construction of gauge theories. In this section, we will first review the most basic example of a gauge theory based on an indecomposable representation, Gupta-Bleuler electrodynamics. We will then see how the indefinite harmonic construction allows twistors to be viewed as a gauge theory of the conformal group in this sense. Finally, we will attempt to construct a Gupta-Bleuler triplet for twistors.

A. Gupta-Bleuler Electrodynamics. Maxwell's equations on Minkowski space may be written in terms of differential forms as

$$
\begin{aligned}
& \delta F=J, \\
& d F=0 .
\end{aligned}
$$

Equation (3.1a) implies current conservation, $\delta J=0$; and on topologically trivial domains, (3.1b) implies $F=d A$, where $A$ is determined up to a closed, and thus exact, one form. In other words, $F$ can be expressed in terms of a potential $A$ which has an unphysical gauge degree of freedom characterized by $A=d \Lambda$. In terms of $A$, Eqs. (3.1) become

$$
\delta d A=J,
$$

whose huge kernel is a manifestation of the gauge degree of freedom. In order to construct a quantum theory, this gauge degree of freedom must be controlled, so instead of (3.2), one considers

$$
\square A=(\delta d+d \delta) A=J
$$

(this corresponds to adding a gauge fixing term, in the Feynman gauge, to the Lagrangian). When the Lorentz condition, $\delta A=0$, is imposed, (3.3) yields Maxwell's equations in the Lorentz gauge. There is still a residual gauge degree of freedom given by $A=d \Lambda$, where $\Lambda$ must satisfy

$$
\delta d \Lambda=0 .
$$


Note that current conservation implies

$$
\delta d(\delta A)=0 .
$$

In group theoretic language, we have the following. Denote

$$
\begin{aligned}
& X_{3}=\{A: \square A=0\}, \\
& X_{2}=\{A: \square A=0, \delta A=0\}, \\
& X_{1}=\{A: A=d \Lambda, \delta A=0\} .
\end{aligned}
$$

These three spaces form a sequence, $X_{3} \supseteq X_{2} \supseteq X_{1}$, of invariant subspaces, none of which is invariantly complemented. In other words, we have an indecomposable representation of the Poincare group in which $X_{1}$ carries the irreducible representation $D(0,0)$, which corresponds to the longitudinal, or gauge, photons; $X_{2} / X_{1}$ carries the representation $D(0,1)+D(0,-1)$, which corresponds to the transverse, or physical, photons; and $X_{3} / X_{2}$ carries the representation $D(0,0)$, which corresponds to time-like, or scalar photons. This structure is known as the Gupta-Bleuler triplet. Note that (3.4) and (3.5) imply that both the gauge and scalar modes propagate as free fields.

B. Twistors as a Gauge Theory. One of the basic results of twistor theory is a correspondence between algebraic and complex analytic data on $\mathbb{P} \mathbb{C}^{3}$ and solutions of the massless free field equations on Minkowski space (see [We] for a mathematical discussion of twistors as well as for the original references). In one formulation, the Penrose transformation maps $H^{0,1}\left(\mathbb{P} \mathbb{T}^{+}, \mathbf{H}^{m}\right)$ to positive frequency fields of helicity $j$, where

$$
m=-2 j-2
$$

and $\mathbb{P} \mathbb{T}^{+}$is positive projective twistor space. As a homogeneous space, $\mathbb{P} \mathbb{T}^{+}$is equivalent to $S U(2,2) / S(U(1) \times U(1,2))$, and glancing at $(2.11)$, the connection to the indefinite harmonic construction is immediate. In particular, we have a partial Gupta-Bleuler structure

$$
\begin{aligned}
& X_{2}=\left\{\phi \in L_{2}^{0,1}(G / H): \bar{\partial} \phi=0, \bar{\partial}^{*} \phi=0\right\}=\mathscr{H}_{2}^{1}\left(G / H, \mathbf{H}^{m}\right), \\
& X_{1}=\left\{\phi \in L_{2}^{0,1}(G / H): \phi=\bar{\partial} \Lambda, \bar{\partial}^{*} \phi=0\right\} .
\end{aligned}
$$

As Dolbeault cohomology classes correspond to field strengths in Minkowski space, the identification of the Dolbeault cohomology with the quotient $X_{2} / X_{1}$ allows the Dolbeault coboundaries, representing the twistor "gauge" degree of freedom, to be interpreted as conformal gauge fields. Positive frequency, negative helicity fields may be obtained in a similar manner by identifying $\mathbb{P P}^{*-}$ with $S U(2,2) / S(U(2,1) \times U(1))$ and using twistor duality [EG].

For the case of helicity one, electromagnetism, the gauge subspace is $D\left(3, \frac{1}{2}, \frac{1}{2}\right)$ (from (2.12)). Note that in Dirac's work [D1], which deals with field strengths, the gauge subspace is also $D\left(3, \frac{1}{2}, \frac{1}{2}\right)$; but in [BFH1], which deals with potentials on the projective cone, the gauge subspace is $D\left(1, \frac{1}{2}, \frac{1}{2}\right)$. This is certainly not surprising as in twistor theory, Dolbeault classes correspond to field strengths and not to vector potentials. 
Viewing twistors as a gauge theory based on an indecomposable representation of the conformal group also brings out a similarity with Dirac singleton theory [FF1-6]. Singletons are a gauge theory based on an indecomposable representation of the de Sitter group, and here the gauge structure is a global phenomenon. In other words, locally, all modes are gauge, and consequently all of the physics resides on the boundary at spatial infinity. Likewise, if one considers the structure (3.8) locally, say on a polydisc, then by the $\bar{\partial}$-Poincare Lemma, all closed forms are exact; and thus locally, all modes are gauge.

C. Ambitwistors. The structure of (3.8), however, is inadequate for a full GuptaBleuler program. There, as in twistor theory, the self-dual and anti-self-dual cases are treated separately, thus precluding the introduction of currents. To overcome this limitation, the twistor program has been generalized to ambitwistors, the basis of which is ambitwistor space,

$$
\mathbb{A}=\left\{\left(L_{1}, L_{3}\right) \in \mathbb{P} \mathbb{T} \times \mathbb{P} \mathbb{T}^{*}: L_{1} \subset L_{3}\right\} .
$$

As ambitwistors are probably not as familiar as twistors, we will briefly outline the program, and for a full treatment, the reader should consult [P]. $A$ is a fivedimensional complex hypersurface in $\mathbb{P T} \times \mathbb{P T}^{*}$ and corresponds to null lines in compactified, complexified Minkowski space, $\mathbb{M}$. For a "nice" Stein domain $U \subseteq \mathbb{M}$, the corresponding region in $\mathbb{A}$ is denoted by $L(U)$. There are natural projections

$$
\pi_{1}: \mathbb{A} \rightarrow \mathbb{P} \mathbb{T}, \quad \pi_{3}: \mathbb{A} \rightarrow \mathbb{P} \mathbb{T}^{*},
$$

where $\pi_{i}\left(L_{1}, L_{3}\right)=L_{i}$. Using these projections, line bundles on $\mathbb{P} \mathbb{T}$ and $\mathbb{P} \mathbb{T}^{*}$ can be pulled back to $\mathbb{A}$; and the pull-backs of the hyperplane bundles are denoted by $\mathcal{O}(m, n)$. In generalization of the twistor program, cohomology classes in $H^{0,1}(L(U)$, $\mathcal{O}(m, m)), m \geqq-1$, correspond to general massless fields on $U$ with $m=2 j-2$. It should be noted, though, that the ambitwistor formulation uses the dual twistor representation [EG], in which cohomology classes are mapped not to field strengths, but to equivalence classes of potentials modulo gauge transformations. In other words, cohomology classes transform into potentials which are determined only up to a Minkowski space gauge transformation.

Many results have been obtained with ambitwistors. Currents have been introduced for the Maxwellian system by [G], using a procedure which generalizes to arbitrary spin. The $\phi^{4}$ equation [H] as well as interacting Yang-Mills fields have also been written in ambitwistor space. For Yang-Mills fields, it has been determined that the axial Yang-Mills current represents the obstruction to extending to a third order neighborhood in the ambient space $\mathbb{P} \mathbb{T} \times \mathbb{P} \mathbb{T}^{*}[\mathrm{HM}]$; and this obstruction can be represented by an inhomogeneous Cauchy-Riemann equation on a cohomological level $([\mathrm{K}, \mathrm{MK}])$. It is interesting that in $[\mathrm{Ha}]$, it has also been found that in order to introduce interactions for conformal Yang-Mills, one must go off the projective cone in $2+4$ conformal space.

For a general Yang-Mills field, the inhomogeneous Cauchy-Riemann equation in ambitwistor space will involve a non-trivial $\bar{\partial}$-connection, so we will for now fix our attention on the abelian case. This corresponds to the Maxwellian system, 
and the inhomogeneous Cauchy-Riemann equation has the form

$$
\bar{\partial} \phi=J
$$

where $\phi \in H^{0,1}(L(U), \mathcal{O}(0,0)), J \in H^{0,2}(L(U), \mathcal{O}(-3,-3))$, and the equation is understood to hold on a third order neighborhood of $L(U)$ in $\mathbb{P} \mathbb{T} \times \mathbb{P} \mathbb{T}^{*}$. Equation (3.9) together with $\bar{\partial}^{*} \phi=0$ give a pair of equations with the same form as Maxwell's equations (3.1), and this seems to point toward a Gupta-Bleuler program that centers around the inhomogeneous Cauchy-Riemann equation.

D. The Gupta-Bleuler Program. From the above considerations, the problem of constructing a twistorial Gupta-Bleuler program can be viewed in two steps. First, determine the triplet on $\mathbb{P} \mathbb{T} \times \mathbb{P} \mathbb{T}^{*}$ or, more appropriately from a group theoretic point of view, on $\mathbb{P} \mathbb{T}^{+} \times \mathbb{P T}^{*-}$; and then second, determine how to pass to $A$. With the first step in mind, let us briefly recall the solution of the inhomogeneous Cauchy-Riemann equation in the case of a Hermitian (positive definite) complex manifold. For forms of degree greater than zero, (3.9) is an overdetermined system of partial differential equations; and one has the consistency requirement that $\bar{\partial} J=0$. This equation also has a large kernel consisting of forms satisfying $\bar{\partial} \phi=0$, so one thus looks for a canonical solution orthogonal to the kernel of $\bar{\partial}$. Hodge theory gives the orthogonal decomposition

$$
\phi=\bar{\partial} \Lambda+\bar{\partial}^{*} \theta+\omega,
$$

where $\omega$ is harmonic, so the canonical solution lies in the image of $\bar{\partial}^{*}$. One thus considers the equation

$$
\bar{\partial} \bar{\partial}^{*} \theta=J
$$

or for general $J$, the elliptic equation

$$
\square \theta=\left(\bar{\partial} \bar{\partial}^{*}+\bar{\partial}^{*} \bar{\partial}\right) \theta=J .
$$

Note that if in (3.12), $\bar{\partial} J=0$, then $\bar{\partial} \bar{\partial}^{*} \bar{\partial} \theta=0$, so $\left\langle\bar{\partial} \bar{\partial}^{*} \bar{\partial} \theta, \bar{\partial} \theta\right\rangle=\left\langle\bar{\partial}^{*} \bar{\partial} \theta, \bar{\partial}^{*} \bar{\partial} \theta\right\rangle=0$, and thus $\bar{\partial}^{*} \bar{\partial} \theta=0$. In this case, (3.12) is equivalent to (3.11). Equation (3.12) is actually a subtle boundary problem in disguise (having to do with defining the Hilbert space analogue of the formal adjoint $\bar{\partial}^{*}$ ), and it has been shown that for certain domains, there is a unique solution of the form $\phi=\bar{\partial}^{*} \theta$ to (3.9) (see [FK] for a full treatment).

Although this is no longer exactly true in the indefinite case, there is an interesting parallel with the Gupta-Bleuler formalism. Equation (3.11) has the same form as Maxwell's equations for the potential, and (3.12) corresponds to the wave equation resulting from gauge fixing. This motivates us to define (at least locally as $\left.\bar{\partial}^{*} . \phi=0\right)$ an ambitwistor potential $\theta$, where $\theta$ is a $(0,2)$-form such that $\bar{\partial}^{*} \theta=\phi$ (this potential is not to be confused with the ordinary potential in Minkowski space). However, as the metric is indefinite, $\bar{\partial} J=0$ (current conservation) no longer implies the equivalence of (3.11) and (3.12). In order to recover solutions to (3.11) from (3.12), we must impose a "Lorentz condition" $\bar{\partial} \theta=0$.

The indefiniteness of the metric also implies that the Hodge decomposition (3.10) is no longer true. Just as in Minkowski space electrodynamics, there is a 
residual gauge degree of freedom even after gauge fixing. Formally, on the space of ambitwistor potentials, a candidate for a Gupta-Bleuler triplet is the following:

$$
\begin{aligned}
& X_{3}=\{\theta: \square \theta=0\}, \\
& X_{2}=\{\theta: \square \theta=0, \bar{\partial} \theta=0\}, \\
& X_{1}=\left\{\theta: \theta=\bar{\partial}^{*} \alpha, \bar{\partial} \theta=0\right\} .
\end{aligned}
$$

But in spite of this formal analogy, there is a serious drawback in the physical interpretation of (3.13). In the positive definite case, $\bar{\partial} \bar{\partial}^{*} \theta=0$ implies $\bar{\partial}^{*} \theta=0$, and thus that $\phi=0$. In the indefinite case, this is no longer true: $\bar{\partial} \bar{\partial}^{*} \theta=0$ implies only that $\bar{\partial}^{*} \theta$, and thus $\phi$, has zero norm. So whereas in the positive definite case the potential determines a unique canonical solution, $\phi=\bar{\partial}^{*} \theta$, to the inhomogeneous Cauchy-Riemann equation, in the indefinite case the potential determines a solution only up to a harmonic form (i.e. both $\bar{\partial}$ and $\bar{\partial}^{*}$ closed) of zero norm. This is the same type of behavior as in the self-dual case on ordinary twistor space, where, by the indefinite harmonic construction of [RSW], each Dolbeault class has a harmonic representative determined only up to a zero norm harmonic form. As a result, the quotient $X_{2} / X_{1}$ corresponds to zero norm field strengths. In other words, it represents the conformal gauge fields on the field strength level, which are completely unphysical.

Therefore, we abandon the ambitwistor potential and attempt to formulate the Gupta-Bleuler triplet on the level of field strengths. To this end, it is natural to consider "weak" harmonic forms satisfying

$$
\square \phi=0 .
$$

In order to maintain the interpretation of currents as $(0,2)$-forms, the inhomogeneous equation should be taken to be

$$
\square \phi=\bar{\partial}^{*} J .
$$

However, to recover (3.9), applying the Lorentz condition $\bar{\partial}^{*} \phi=0$ is not enough as it yields $\bar{\partial}^{*} \bar{\partial} \phi=\bar{\partial}^{*} J$, or equivalently

$$
\bar{\partial} \phi=J+\gamma,
$$

where $\gamma$ is an arbitrary zero norm, harmonic $(0,2)$-form. But this is not really a problem, for as all zero norm quantities are unphysical, we can take physical currents as equivalence classes of closed $(0,2)$-forms modulo zero norm harmonic forms. We now have as a candidate for the Gupta-Bleuler triplet the following:

$$
\begin{aligned}
& X_{3}=\{\phi: \square \phi=0\}, \\
& X_{2}=\left\{\phi: \bar{\partial} \phi=0, \bar{\partial}^{*} \phi=0\right\}, \\
& X_{1}=\left\{\phi: \phi=\bar{\partial} \Lambda, \bar{\partial}^{*} \phi=0\right\} .
\end{aligned}
$$

Initially, we may consider $\phi$ to be a smooth compactly supported form in $E^{0,1}\left(\mathbb{P} \mathbb{T}^{+} \times \mathbb{P} \mathbb{T}^{*-}, \mathcal{O}(0,0)\right)$, finite under the $G$-invariant indefinite metric. Then there is no problem in defining the formal adjoint $\bar{\partial}^{*}$, and this should not seriously impair the physical development of the theory. With (3.17), one can consider 
propagation and quantization in the context of ambitwistors. This, as well as completion under an appropriate norm, will be the subject of future work.

There is a lot of motivation for further investigation. The ambitwistor program has the capacity to treat all spins in an equivalent manner, and this suggests the existence of a generalization of the Cauchy-Riemann equation to arbitrary spin. Since interacting Yang-Mills fields have been constructed with ambitwistors, this is especially interesting as it may lead to an interacting theory with higher spins. Furthermore, as points in ambitwistor space correspond to null lines in complex Minkowski space, further investigation may contribute to the already noted connection between ambitwistors and strings [IY, Ho, Sw1, 2].

\section{The de Sitter Group}

One of the most interesting gauge theories based on an indecomposable representation is that of the Dirac singletons [FF1-6]. The representation in question is highly singular, lying in the "Wallach set" of the analytic continuation of the holomorphic discrete series. As the indefinite harmonic construction applies to certain representations in the analytic continuation of the holomorphic discrete series, we will apply it to the de Sitter group in the hope of obtaining a geometric realization of the Dirac singletons.

As before, the first step is to construct the space $G / H$ and verify the holomorphic condition. Let $G=S p(2, \mathbb{R})$. The requirement that $G / H$ be a pseudoHermitian symmetric space requires that $H=U(1,1)[\mathrm{Sr}]$. Note that since all pseudo-Hermitian symmetric spaces are simply connected and in one-to-one correspondence with pseudo-Hermitian Lie algebras, it is sufficient to deal with $S p(2, \mathbb{R})$. Thus

$$
\begin{aligned}
\mathfrak{g}_{0} & =\mathfrak{s p}(2, \mathbb{R}), \\
\mathfrak{h}_{0} & =\mathfrak{u}(1,1), \\
\mathfrak{I}_{0} & =\mathfrak{u}(2), \\
\mathfrak{m}_{0} & =\mathfrak{s u}(1,1)+\mathfrak{s u}(1,1), \\
\mathfrak{l}_{0} & =\mathfrak{u}(1)+\mathfrak{u}(1) .
\end{aligned}
$$

Parametrize $\mathfrak{g}_{0}$ in the following manner:

Then

$$
\mathfrak{g}_{0}=\left\{\left[\begin{array}{cccc}
i \theta_{1} & a & c & b \\
-\bar{a} & i \theta_{2} & b & d \\
\bar{c} & \bar{b} & -i \theta_{1} & \bar{a} \\
\bar{b} & \bar{d} & -a & -i \theta_{2}
\end{array}\right]: \theta_{i} \in \mathbb{R} ; a, b, c, d, \in \mathbb{C}\right\} .
$$

$$
\mathfrak{h}_{0}=\left\{\left[\begin{array}{cccc}
i \theta_{1} & 0 & 0 & b \\
0 & i \theta_{2} & b & 0 \\
0 & \bar{b} & -i \theta_{1} & 0 \\
\bar{b} & 0 & 0 & -i \theta_{2}
\end{array}\right]: \theta_{i} \in \mathbb{R}, b \in \mathbb{C}\right\}
$$




$$
\begin{aligned}
& \mathfrak{f}_{0}=\left\{\left[\begin{array}{cccc}
i \theta_{1} & a & 0 & 0 \\
-\bar{a} & i \theta_{2} & 0 & 0 \\
0 & 0 & -i \theta_{1} & \bar{a} \\
0 & 0 & -a & -i \theta_{2}
\end{array}\right]: \theta_{i} \in \mathbb{R}, a \in \mathbb{C}\right\} \\
& \mathfrak{q}_{0}=\left\{\left[\begin{array}{cccc}
0 & a & c & 0 \\
-\bar{a} & 0 & 0 & d \\
\bar{c} & 0 & 0 & \bar{a}
\end{array}\right]: a, c, d \in \mathbb{C}\right\} \\
& \begin{aligned}
& \mathfrak{p}_{0}=\{ {\left.\left[\begin{array}{llll}
0 & 0 & c & b \\
0 & 0 & b & d \\
\bar{c} & \bar{b} & 0 & 0 \\
\bar{b} & \bar{d} & 0 & 0
\end{array}\right]: b, c, d \in \mathbb{C}\right\} } \\
& \mathfrak{m}_{0}=\left\{\left[\begin{array}{cccc}
i \theta_{1} & 0 & c & 0 \\
0 & i \theta_{2} & 0 & d \\
\bar{c} & 0 & -i \theta_{1} & 0 \\
0 & \bar{d} & 0 & -i \theta_{2}
\end{array}\right]: c, d \in \mathbb{C}, \theta_{i} \in \mathbb{R}\right\}
\end{aligned} \\
& \mathfrak{l}_{0}=\left\{\left[\begin{array}{cccc}
i \theta_{1} & 0 & 0 & 0 \\
0 & i \theta_{2} & 0 & 0 \\
0 & 0 & -i \theta_{1} & 0 \\
0 & 0 & 0 & -i \theta_{2}
\end{array}\right]: \theta_{i} \in \mathbb{R}\right\} \text {. }
\end{aligned}
$$

With respect to our parametrization, let us note the following:

(1) The Cartan decomposition $\left(\mathfrak{g}_{0}, \theta\right)$ is unique, and thus the Cartan decomposition is invariant under $\operatorname{Ad}_{G}(K)$.

(2) The pseudo-Hermitian Lie algebra $\left(g_{0}, \tau\right)$ is unique up to conjugation under $\operatorname{Ad}_{G}(K)$.

Proof. (1) This is obvious by inspection. (2) Since $\left(\mathfrak{g}_{0}, \tau\right)$ is pseudo-Hermitian, $\mathfrak{h}_{0}$ is the $\mathfrak{g}_{0}$ centralizer of a $\mathfrak{u}(1)$ subalgebra, $t_{0} . t_{0} \subseteq \mathfrak{s} \mathfrak{u}(2)$, otherwise $\mathfrak{h}_{0}=\mathfrak{u}(2) . t_{0}$ is maximal compact in $\mathfrak{s u}(2)$, and thus all such $\mathrm{t}_{0}$ are conjugate under $\operatorname{Ad}_{G}(K)$. The corresponding $\mathfrak{g}_{0}$ centralizers are thus conjugate, giving the claim.

The central elements giving the almost complex structure (unique up to sign since $G$ is simple) on $G / H$ and $G / K$ respectively are

$$
\zeta=\frac{1}{2}\left[\begin{array}{cccc}
i & 0 & 0 & 0 \\
0 & -i & 0 & 0 \\
0 & 0 & -i & 0 \\
0 & 0 & 0 & i
\end{array}\right] \text { and } \eta=\frac{1}{2}\left[\begin{array}{cccc}
i & 0 & 0 & 0 \\
0 & i & 0 & 0 \\
0 & 0 & -i & 0 \\
0 & 0 & 0 & -i
\end{array}\right]
$$

Direct calculation gives

$$
\mathfrak{q}_{+}=\left\{\left[\begin{array}{cccc}
0 & a & c & 0 \\
0 & 0 & 0 & 0 \\
0 & 0 & 0 & 0 \\
0 & d & -a & 0
\end{array}\right]: a, c, d \in \mathbb{C}\right\}
$$




$$
\mathfrak{p}_{+}=\left\{\left[\begin{array}{llll}
0 & 0 & c & b \\
0 & 0 & b & d \\
0 & 0 & 0 & 0 \\
0 & 0 & 0 & 0
\end{array}\right]: b, c, d \in \mathbb{C}\right\} .
$$

However, this time $\left[\mathfrak{p} \cap \mathfrak{q}_{+},\left[\mathfrak{p} \cap \mathfrak{q}_{+}, \mathfrak{f} \cap \mathfrak{q}_{-}\right]\right] \neq 0$. This is not surprising in view of the fact that $\mathfrak{p}_{-} \cap \mathfrak{q}_{+} \neq 0$. In view of (1) and (2) from above, this result is independent of the specific realization of $\mathfrak{h}_{0}$ within our parametrization. Thus, $\operatorname{Sp}(2, \mathbb{R}) / U(1,1)$ does not satisfy the holomorphic condition necessary to apply the indefinite harmonic construction of [RSW].

But this does not mean that the indefinite harmonic construction is completely inapplicable to the de Sitter group. By restricting the massless representations of $S U(2,2)$, one may obtain the massless representations of the de Sitter group [AFFS]. The Dirac singletons, however, are not realized in this context [AFFS].

\section{Conclusion}

In this note, we have taken a very elegant mathematical construction and examined some potential physical applications. First, we explicitly constructed the indefinite harmonic representations in the case of $S U(2,2)$ and established the relationship to the minimal energy representations as obtained in the physics literature. We then saw that the indefinite harmonic construction allows one to view twistors as a gauge theory of the conformal group. In particular, it is now clear that the coboundaries of the twistor cohomology represent a gauge degree of freedom, on the level of field strengths, associated with an invariant subspace of an indecomposable representation of the conformal group. With this point of view, we also noted a similarity between twistors and the Dirac singletons.

However, as the self-dual and anti-self-dual cases are treated separately, the basic construction is not suitable for a full Gupta-Bleuler program. Ambitwistors appear to provide the basis for a generalization. In analogy with Minkowski space electrodynamics, one can formally define an ambitwistor potential which, in ambitwistor space, parallels the vector potential in Minkowski space. There is formally a Gupta-Bleuler triplet for the ambitwistor potential, but its physical interpretation is trivial. We thus attempted to construct a Gupta-Bleuler triplet on the level of field strengths, arriving at (3.17). Further investigation, though, is necessary; and this seems completely justified in view of the capacity to treat higher spin fields uniformly as well as the already noted connection with strings.

Lastly, we attempted to apply the indefinite harmonic construction to the de Sitter group but found that the indefinite harmonic construction is not directly applicable to the de Sitter group or any of its covers. One may still obtain the massless representations by restricting the massless representations of $S U(2,2)$, but contrary to claims in the literature, the singleton representations are not realized in this geometric context.

Acknowledgements. The author would like to thank C. Fronsdal and M. Flato for suggesting this problem as well as for their advice and criticism. He would also like to thank A. Haidari for reading this manuscript and for many interesting conversations on conformal theories. This work was supported in part by the National Science Foundation under grant \#PHY-86-13201. 


\section{References}

[A] Araki, H.: Indecomposable representations with invariant inner product. Commun. Math. Phys. 97, 149-159 (1985)

[AFFS] Angelopoulos, E., Flato, M., Fronsdal, C., Sternheimer, D.: Massless particles, conformal group, and de Sitter universe. Phys. Rev. D 23, 1278-1289 (1981)

[BFH1] Binegar, B., Fronsdal, C., Heidenreich, W.: Conformal QED. J. Math. Phys. 24, 2828-2846 (1983)

[BFH2] Binegar, B., Fronsdal, C., Heidenreich, W.: de Sitter QED. Ann. Phys. 149, 254-272 (1983)

[D1] Dirac, P. A. M.: Wave equations in conformal space. Ann. Math. 37, 429-442 (1936)

[D2] Dirac, P. A. M.: A remarkable representation of the $3+2$ de Sitter group. J. Math. Phys. 4, 901-909 (1963)

[EG] Eastwood, M. G., Ginsberg, M. L.: Duality in twistor theory. Duke Math. J. 48, 177-196(1981)

[FF1] Flato, M., Fronsdal, C.: One massless particle equals two Dirac singletons. Lett. Math. Phys. 2, 421-426 (1978)

[FF2] Flato, M., Fronsdal, C.: On dis and racs. Phys. Lett. 97B, 236-240 (1980)

[FF3] Flato, M., Fronsdal, C.: Quantum field theory of singletons. The Rac. J. Math. Phys. 22, 1100-1105 (1981)

[FF4] Flato, M., Fronsdal, C.: Quarks or singletons? Phys. Lett. B 172, 412-416 (1986)

[FF5] Flato, M., Fronsdal, C.: The singleton dipole. Commun. Math. Phys. 108, 469-482 (1987)

[FF6] Flato, M., Fronsdal, C.: How the BRST invariance of QED is induced from the underlying singleton field theory. Phys. Lett. B 189, 145-148 (1987)

[FK] Folland, G., Kohn, J.: The Neumann problem for the Cauchy-Riemann complex. Princeton, NJ: Princeton University Press 1972

[F] Fronsdal, C.: Semisimple gauge theories and conformal gravity. In: Applications of group theory in physics and mathematical physics. Flato, M., Sally, P., Zuckerman, G. (eds.). Lect. in App. Math. Vol. 21. Providence, R.I.: American Mathematical Society 1985

[FPS] Furlan, P., Petkova, V. B., Sotkov, G. M.: Indecomposable representations of the conformal group: A nonsingular Photon-Weyl graviton system. J. Math. Phys. 28, 251-257 (1987)

[G] Gindikin, S. G.: Twistor representation of solutions of a Maxwellian system. Funk. Anal. Pril. 14, 64-66 (1980). English translation: Funct. Anal. Appl. 14, 298-300 (1981)

[Ha] Haidari, A.: Conformal quantum Yang-Mills. J. Math. Phys. 27, 2409-2414 (1986)

[H] Henkin, G. M.: Representation of solutions of the $\phi^{4}$ equation in the form of holomorphic vector bundles over twistor space. Sov. Math. Dok1. 24, 415-419 (1981)

[HM] Henkin, G. M., Manin, Yu. I.: Twistor description of classical Yang-Mills-Dirac fields. Phys. Lett. 95B, 405-408 (1980)

[Ho] Horowitz, G.: Introduction to string theories. Santa Babara preprint, 1985

[IY] Isenberg, J., Yasskin, P.: Ambitwistors (and Strings?). In: Proceedings of the Oregon Meeting, Annual Meeting of the Division of Particles and Fields of the American Physical Society. Hwa, R. (ed.). Singapore: World Scientific 1986

[J] Jakobsen, H.: Conformal covariants. Publ. RIMS, Kyoto Univ. 22, 345-364 (1986)

[K] Khenkin, G. M.: Represenation of the Yang-Mills equations in the form of Cauchy-Riemann equations in twistor space. Sov. Phys. Dokl. 25, 986-988 (1980)

[MK] Manin, Yu. I., Khenkin, G. M.: Yang-Mills equations as Cauchy-Riemann equations in twistor space. Sov. J. Nucl. Phys. 35, 941-950 (1982)

[P] Pool, R.: Yang-Mills Fields and extension theory. Memoirs of the Am. Math. Soc. 67, No. 358 (1987)

[RSW] Rawnsley, J., Schmid, W., Wolf, J.: Singular unitary representations and indefinite harmonic theory. J. Funct. Anal. 51, 1-114 (1983)

[Sr] Shapiro, R.: Pseudo-Hermitian symmetric spaces. Commun. Math. Helv. 46, 529-548 (1971)

[Sw1] Shaw, W.: An ambitwistor description of bosonic or supersymmetric minimal surfaces and strings in four dimensions. Class. Quan. Grav. 3, 753-761 (1986)

[Sw2] Shaw, W.: Twistor quantization of open strings in three dimensions. Gen. Rel. Grav. 4, 1193-1205 (1987)

[We] Wells, R.: Complex manifolds and mathematical physics. Bull. Am. Math. Soc. 1, 296-336 (1979) 
[W1] Wolf, J.: Geometric quantization in the spirit of Gupta and Bleuler. In: Differential geometric methods in mathematical physics. Sternberg, S. (ed.). Mathematical Physics Studies, Vol. 6. Dordrecht, Holland: D. Reidel 1984

[W2] Wolf, J.: Indefinite harmonic theory and unitary representations. In: Applications of group theory in physics and mathematical physics. Flato, M., Sally, P., Zuckerman, G. (eds.). Lectures in Applied Mathematics. Vol. 21. Providence, R.I.: American Mathematical Society 1985

Communicated by H. Araki

Received November 18, 1987 
